\title{
Frequency of Cholestatic Liver Diseases in Zagazig University Hospitals, with Special Emphasize on Extrahepatic Causes
}

\author{
Ghada. A. Salem and Ahmad. S. Sherbini \\ Tropical Medicine Department,Faculty of Medicine, Zagazig University, Egypt
}

Corresponding Author: Ahmad S Sherbini

E mail: ahmadsakr65@yahoo. com

Key words: extrahepatic cholestasis; cholidocholithiasis; cancer head of pancreas, Ultrasonography; ERCP
Background and study aim: Extrahepatic cholestasis results from the mechanical obstruction of large bile ducts outside the liver or within the porta hepatis. The common etiologies of cholestasis have been reported to vary from one center to another. Our aim was to assess the frequency of cholestatic disorders in patients admitted to our department and try to identify the underlying extrahepatic causes aiming at improving the quality of management offered to this patients group.

Patients and Methods: In the present study, 506 cases admitted to our department were included. Sixty one of them met our inclusion criteria, of high serum alkaline phosphatase $(A L P \geq 1.5$ times the upper limit of normal), high gamma glutamyl transferase (GGT $\geq 3$ times the upper limit of normal) and ultrasonogrpahic (US) features of extrahepatic cholestasis (dilated intra and/or extra-hepatic ducts) of whatever etiology. The selected patients underwent the following: Full history taking and thorough physical examination, complete blood picture, liver and kidney function tests and abdominal CT. Also, ERCP was performed whenever needed and the tumor markers, alpha feto-protein \& (CA 19-9) were assessed in selected cases "when mass lesions were detected".

Results: The frequency of cholestatic liver diseases presented with elevated both ALP \& GGT as described above, was $\% 19.7$ (100 of 506 patients). While the frequency of extrahepatic cholestasis was $12.1 \%$ with females accounting for $54.1 \%$ and males were $45.9 \%$ with mean ages \pm $\mathrm{SD}$ of $51.1 \pm 11.7$ years. Benign causes of

\section{INTRODUCTION}

Cholestasis is an impairment of bile formation and/or bile flow which may clinically present with fatigue, pruritus and, in its most overt form, jaundice [1]. Cholestatic jaundice is often accompanied by a broad spectrum of laboratory, clinical and histological abnormalities. Early surgical obstructive jaundice were more frequent than malignant ones $(57.4 \% \mathrm{VS}$ $42.6 \%$ ). The most common cause was choledocholithiasis in 25/61 (40.9\%), followed by cancer head of pancreas $9 / 61$ (14.8\%), peri-ampullary carcinoma $8 / 61$ (13.11\%), cholangiocarcinoma 5/61 (8.2\%) and pancreatitis 4/61 (6.6\%). Dark urine, clay stool and itching were more common in patients with malignant obstructive jaundice. There was a highly positive agreement between ALP and GGT with $\mathrm{P}<0.01$. The sensitivity of $\mathrm{U} / \mathrm{S}$, $\mathrm{CT}$ and ERCP in detection of CBD stones were $76 \%, 88 \%$ and $100 \%$ respectively. While in detection of cancer head of pancreas, it was $66.7 \%, 100 \%$ and $100 \%$ respectively. The predictive values of US compared to ERCP in detection of CBD stones was $100 \%$ positive and $85.7 \%$ negative, while the predictive value of US compared to ERCP in detection of cancer head of pancreas was $100 \%$ positive and $94.5 \%$ negative. The predictive values of CT compared to ERCP in detection of CBD stones was $100 \%$ positive and 92.3\% negative, while the predictive value of CT compared to ERCP in detection of cancer head of pancreas was $100 \%$ positive.

Conclusion: We concluded that, the frequency of cholestatic liver diseases in our department was \%19.7. Among them, extrahepatic causes had a frequency of $12.1 \%$ with choledocholithiasis as the commonest benign etiology, while cancer head of pancreas was the most frequently met with malignancy. ERCP is considered the gold standard modality in diagnosis and management of extrahepatic cholestasis.

biochemical markers in asymptomatic patients include elevated serum alkaline phosphatase (ALP) and gamma glutamyl transpeptidase (GGT) followed by conjugated hyperbilirubinemia at more advanced stages [2]. 
Cholestatic liver diseases are characterized by accumulation of hepatotoxic substances, mitochondrial dysfunction and impairment of liver antioxidant defenses. The storage of hydrophobic bile acids has been incriminated as the main cause of hepatotoxicity with alteration of some important cell functions, such as the mitochondrial energy production leading to development of oxidative damage [3].

Moreover, the absence of bile salts from the intestine can produce malabsorption, leading to steatorrhea and deficiencies of fat-soluble vitamins (particularly A,D and K). Vitamin K deficiency leads to prolonged prothrombin time. In long-standing cholestasis, concomitant vitamin D and $\mathrm{Ca}$ malabsorption can cause osteoporosis or osteomalacia [4].

Also in obstructive jaundice, increased intestinal permeability has been postulated to be a key factor contributing to bacterial and endotoxins translocation to mesenteric lymph nodes, portal circulation and the liver. A suppressed clearance capacity of Kupffer cells, the main hepatic macrophage population, attributed to accumulation of bile acids into the liver, permits the "spillover" of endotoxins from portal into systemic circulation, with consecutive release of pro-inflammatory cytokines, potentially leading to the development of the so called "gut derived sepsis" [3,22].

Nevertheless, the first critical step in approach to patients with cholestatic liver diseases, is to differentiate intra- and extra-hepatic cholestasis, bearing in mind that several intra-hepatic causes of cholestatic jaundice can mimic extra-hepatic obstruction to varying degree [1].

Jaundice due to biliary obstruction may be caused by a heterogeneous group of diseases that include both benign and malignant conditions. Benign causes include choledocholithiasis, primary sclerosing cholangitis, Mirrizi syndrome, postoperative billiary stricture, post inflammatory stricture, pancreatitis, choledochal cyst, recurrent pyogenic cholangitis, parasitic diseases, duodenal diverticulosis and AIDS cholangiopathy [5].

While malignant causes include cancer head of pancreas, carcinoma of the gall bladder, cholangiocarcinoma, carcinoma of the duodenum, ampullary tumors, hepatocellular carcinoma, lymphoma and metastatic tumors [6].

Extrahepatic cholestasis may be additionally subdivided into intraductal and extraductal.
Intraductal obstruction is caused by neoplasms, stone disease, biliary stricture, parasites, primary sclerosing cholangitis (PSC), AIDS-related cholangiopathy, and biliary tuberculosis. While extraductal obstruction is caused by external compression of the biliary ducts as that secondary to neoplasms, pancreatitis, or cystic duct stones with subsequent gallbladder distension [7].

In hepatic parenchymal diseases (e.g. cirrhosis), fibrosis may prevent the intrahepatic bile ducts dilatation, resulting in dilation confined to the extrahepatic bile ducts. The question then is whether the derangement of liver functions is related to the hepatic or extrahepatic etiology [8]. The common cause of obstructive jaundice have been reported to vary from one center to another.

Our aim was to assess the frequency of cholestatic disorders in patients admitted to our department and try to identify the underlying extrahepatic causes aiming at improving the quality of management offered to this patients group.

\section{PATIENTSAND METHODS}

The present cross sectional study was conducted in Tropical Medicine Department, Zagazig university hospitals during the period from May 2012 to March 2013.

The study was conducted on 506 cases admitted serially to our department and accepted to participate in the present work. They were all subjected to:

1- Serum Bilirubin (total \& direct), ALT, AST and ALP levels were assayed by (colorimetric assay RXL, Semiens, Germany).

2- Serum gamma glutamyl transferase level: (by colorimetric assay, Cobas, Roche, Germany). The inclusion criteria were high serum alkaline phosphatase level $(\geq 1.5$ times the upper limit of normal), high gamma glutamyl transferase ( $\geq 3$ times the upper limit of normal) according to EASL [9].

3- Pelvi abdominal ultrasound by Ezzaoti-Mylab 20. ultrasonographic features of extrahepatic cholestasis included (dilated extra and/or intrahepatic ducts) of whatever cause.

All selected patients underwent the following:

- Full history taking and through physical examination. 
- Assessment of body mass index (BMI) was conducted using the following formula:

$$
\mathrm{BMI}=\frac{\operatorname{mass}(\mathrm{kg})}{(\operatorname{height}(\mathrm{m}))^{2}}
$$

Body mass index (BMI) is defined as the individual's body mass divided by the square of their height with the value universally being given in units of $\mathrm{kg} / \mathrm{m}^{2}$.

- Complete blood count, using sympx K x 21 cell counter (Roche diagnostics Manheim, Germany).

- Tumor markers: cancer antigen (CA 19-9), normal levels <37 U/ml. [10] \& Alpha fetoprotein (AFP): normal range $10-20 \mathrm{ng} / \mathrm{ml}$, while a level $400 \mathrm{ng} / \mathrm{ml}$ is considered diagnostic for $\mathrm{HCC}$ in the presence of suggestive ultrasonography [11].

- Abdominal CT: Triphasic CT scanning of the liver was performed with CTi/Pro GE medical system and Toshiba $\mathrm{X}$-Vision single slicer CT scanner.

- ECRP whenever needed for diagnosis and/or management of extrahepatic cholestasis.

\section{Statistically analysis:}

Data were checked, entered and analyzed using Epi-Info (2000) for data processing and statistics.

\section{Descriptive statistics:}

Data were expressed as numbers and percentages for qualitative variables and mean \pm standard deviation for quantitative one.
Validity of a screening test:

Sensitivity: is the ability of the test to detect true positive cases $=$

$$
\frac{\text { True positive }}{\text { True positive }+ \text { False negative }}
$$

Specificity: is the ability of the test to detect true negative cases $=$

$$
\frac{\text { True negative }}{\text { True negative }+ \text { False positive }}
$$

Positive predictive value: is the probability of disease in a patient with positive test result $=$

True positive

$\overline{\text { True positive }+ \text { False positive }}$

Negative predictive value: is the probability of not having the disease in a subject with negative test result $=$

$$
\frac{\text { True negative }}{\text { True negative }+ \text { False negative }}
$$

Accuracy: is the proportion of all test results both positive and negative that are correct $=$

$$
\frac{\text { True positive }+ \text { True negative }}{\text { True positive }+ \text { True negative }+ \text { False positive }+ \text { False negative }}
$$

\section{RESULTS}

The frequency of cholestatic liver diseases was $19.7 \%$ (100 of 506 patients). While the extrahepatic cholestatic causes were detected in 61 out of 506 studied cases representing $12.1 \%$ being more in middle aged patients and more in females than males as shown in table (1).

Table (1): Demographic data of cases with extrahepatic cholestasis.

\begin{tabular}{|c|c|c|c|c|}
\hline \multirow{2}{*}{} & \multicolumn{2}{|c|}{ Sex } & \multicolumn{2}{c|}{ Age } \\
\cline { 2 - 3 } & Male & Female & Range & Mean \pm SD \\
\hline NO & 28 & 33 & \multirow{2}{*}{$25-72$} & $51.1 \pm 11.7$ \\
\hline$\%$ & 45.9 & 54.1 & & \\
\hline \multicolumn{4}{|c|}{ Total NO = 61 } \\
\hline
\end{tabular}

The majority of patients in our study had benign causes for their obstructive jaundice (57.4\%) with choledocholithiasis as the most common cause comprising $40.9 \%$ of cases. While malignant causes represented $42.6 \%$ of cases with cancer head of pancreas as the commonest malignancy detected, as shown table (2). 
Table (2): Frequency of causes of extrahepatic cholestasis.

\begin{tabular}{|c|c|c|}
\hline Etiology & NO & $\%$ \\
\hline CBD stone & 25 & $40.9 \%$ \\
\hline Cancer head of pancreas & 9 & $14.8 \%$ \\
\hline Periampullary carcinoma & 8 & $13.11 \%$ \\
\hline Cholangiocarcinoma & 5 & $8.2 \%$ \\
\hline Acute pancreatitis & 4 & $6.6 \%$ \\
\hline Porta hepatis Lymphadenopathy & 3 & $4.9 \%$ \\
\hline Cholangitis & 2 & $3.3 \%$ \\
\hline Hepatocellular carcinoma & 2 & $3.3 \%$ \\
\hline Duodenal diverticulum & 1 & $1.6 \%$ \\
\hline Iatrogenic ligation of CBD & 1 & $1.6 \%$ \\
\hline Pancreatic pseudocyst & 1 & $1.6 \%$ \\
\hline Total & 61 & $100 \%$ \\
\hline
\end{tabular}

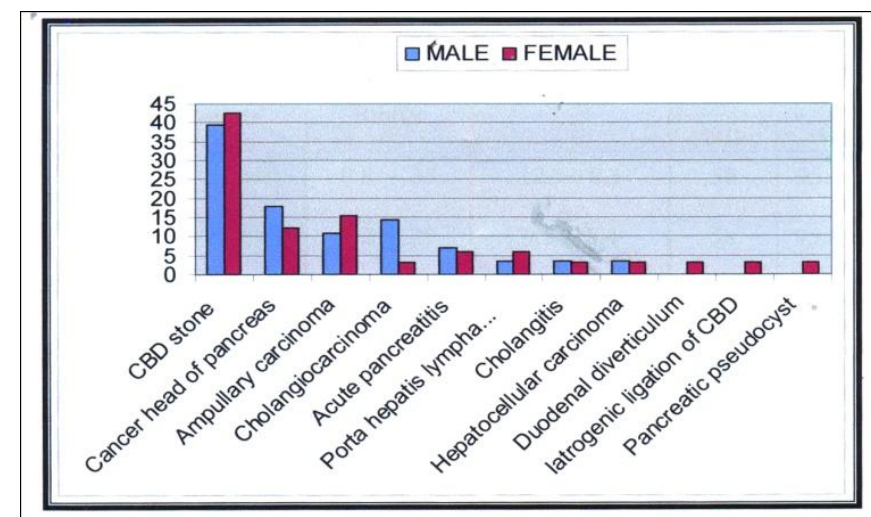

Fig. (1): Relation between etiology of extraheaptic cholestasis and sex

The percentage of malignant extrahepatic cholestasis was higher in males $(50 \%)$ than females (36.4\%) as seen in Figure (1). Most of the patients with benign obstructive jaundice were younger in age as the percentage of benign causes was the highest $(85.7 \%)$ in the $20-40$ years age group, while malignant causes were in elderly as the percentage of malignant causes was the highest $(66.7 \%)$ in the age group of $>60$ years Table (3).

Table (3): Relation between malignant and non malignant causes of extrahepatic cholestasis and age

\begin{tabular}{|c|c|c|c|c|c|c|}
\hline \multicolumn{2}{|l|}{ Age group } & Malignant & Non malignant & Total & $\mathrm{X} 2$ & $\mathbf{P}$ \\
\hline \multirow[t]{2}{*}{$20-40$ (adult) } & NO & 2 & 12 & 14 & \multirow{2}{*}{7.12} & \multirow{2}{*}{0.007} \\
\hline & $\%$ & $14.3 \%$ & $85.7 \%$ & & & \\
\hline \multirow[t]{2}{*}{ 41-60 (middle) } & NO & 16 & 19 & 35 & \multirow{2}{*}{0.76} & \multirow{2}{*}{0.38} \\
\hline & $\%$ & $45.7 \%$ & $54.3 \%$ & & & \\
\hline \multirow[t]{2}{*}{$>60$ (elderly) } & NO & 8 & 4 & 12 & \multirow{2}{*}{18.87} & \multirow{2}{*}{0.001} \\
\hline & $\%$ & $66.7 \%$ & $33.3 \%$ & & & \\
\hline Total & & 26 & 35 & 61 & & \\
\hline
\end{tabular}

Serum total and direct bilirubin levels were elevated in $95.1 \%$ of cases with mean values of 12.5 and 9.3 respectively. There was a significant agreement between ALP and GGT, with P value $<0.01$, while serum transaminases were only mildly elevated in $34.4 \%$ of cases. Regarding the clinical manifestations, 58 cases $(95.1 \%)$ presented with jaundice. The percentage of dark urine, itching and clay stool in malignant cases were $92.3 \%, 73.1 \%$ and $53.8 \%$ respectively, 
compared to $82.8 \%, 62.8 \%$ and $48.6 \%$ respectively, in non malignant conditions. Charcot's triad was more common in benign conditions with a percentage of (40\%) compared to $(23.1 \%)$ in malignant cases.

Table (4): Frequency of clinical presentations of cases with extrahepatic cholestasis

\begin{tabular}{|l|c|c|}
\hline \multicolumn{1}{|c|}{ Clinical presentation } & NO & \% \\
\hline Jaundice & 58 & $95.1 \%$ \\
\hline Dark urine & 53 & $86.9 \%$ \\
\hline Itching & 41 & 67.2 \\
\hline Vomiting & 37 & $60.7 \%$ \\
\hline Clay stool & 31 & $50.8 \%$ \\
\hline Weight loss & 25 & $41 \%$ \\
\hline Hepatomegally & 22 & $36.1 \%$ \\
\hline Charcot triad & 20 & $32.8 \%$ \\
\hline Scratch marks & 16 & $26.2 \%$ \\
\hline Bleeding manifestation & 10 & $16.4 \%$ \\
\hline Palpable abdominal mass & 5 & $8.1 \%$ \\
\hline \multicolumn{2}{|c|}{ Total } & $\mathbf{6 1}$ \\
\hline \multicolumn{2}{|c|}{}
\end{tabular}

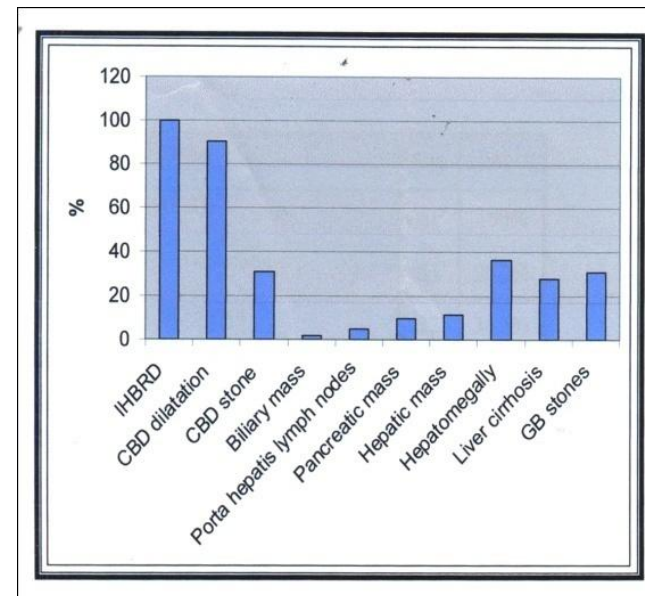

Fig. (2): Frequency of ultrasonographic features of extrahepatic cholestasis

According to imaging modalities ultrasound (U/S) was done to all cases. It picked dilated intrahepatic channels in $100 \%$ of patients of extrahepatic cholestasis and dilated CBD in $90.2 \%$ of them. CBD stones were detected in $31.1 \%$ while masses were detected in only $27.8 \%$ and most of the times, it was in the head of pancreas Figure (2). Abdominal computed tomography (CT) was done to all cases to confirm the diagnosis of (U/S) and/or to establish the cause of obstruction when U/S couldn't detect it. It picked dilated intrahepatic channels in $100 \%$ \& dilated CBD in $90.2 \%$, while CBD stones were detected in $36.1 \%$.
Masses were detected in $41 \%$ of cases Figure (3). Endoscopic retrograde cholangi-pancreatography (ERCP) was done to the cases which were not diagnosed by US or CT or as a therapeutic tool. It could detect all the 25 cases with CBD stones representing $40.9 \%$ of all cases with extrahepatic cholestasis. Cholangiocarcinoma was detected in 5 cases, in which 3 of them showed hilar CBD malignant stricture "Klatskin tumor" and 2 cases showed distal CBD stricture. Portahepatis lymphadenopathy and HCC were demonstrated as either hilar or distal malignant CBD stricture (Figure 4). 


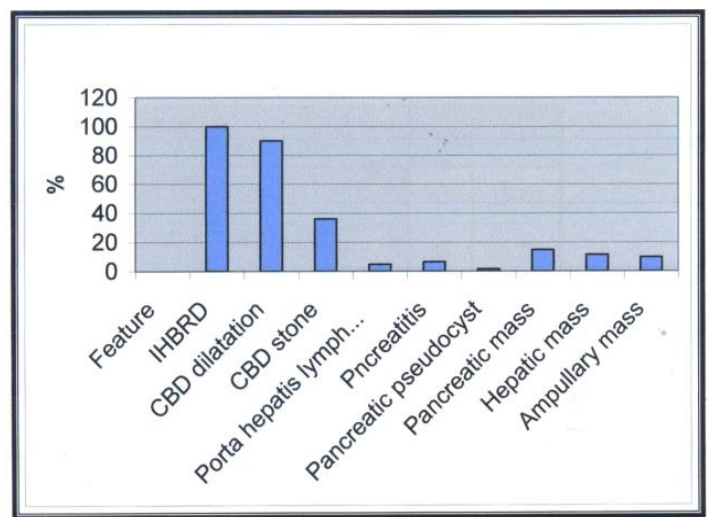

Fig. (4): Frequency of CT features of extrahepatic cholestasis

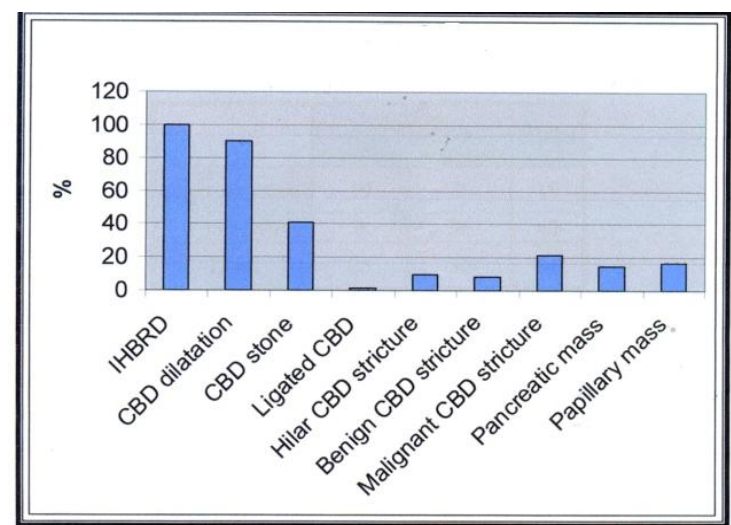

Fig. (5): ERCP features of cases with extrahepatic cholestasis

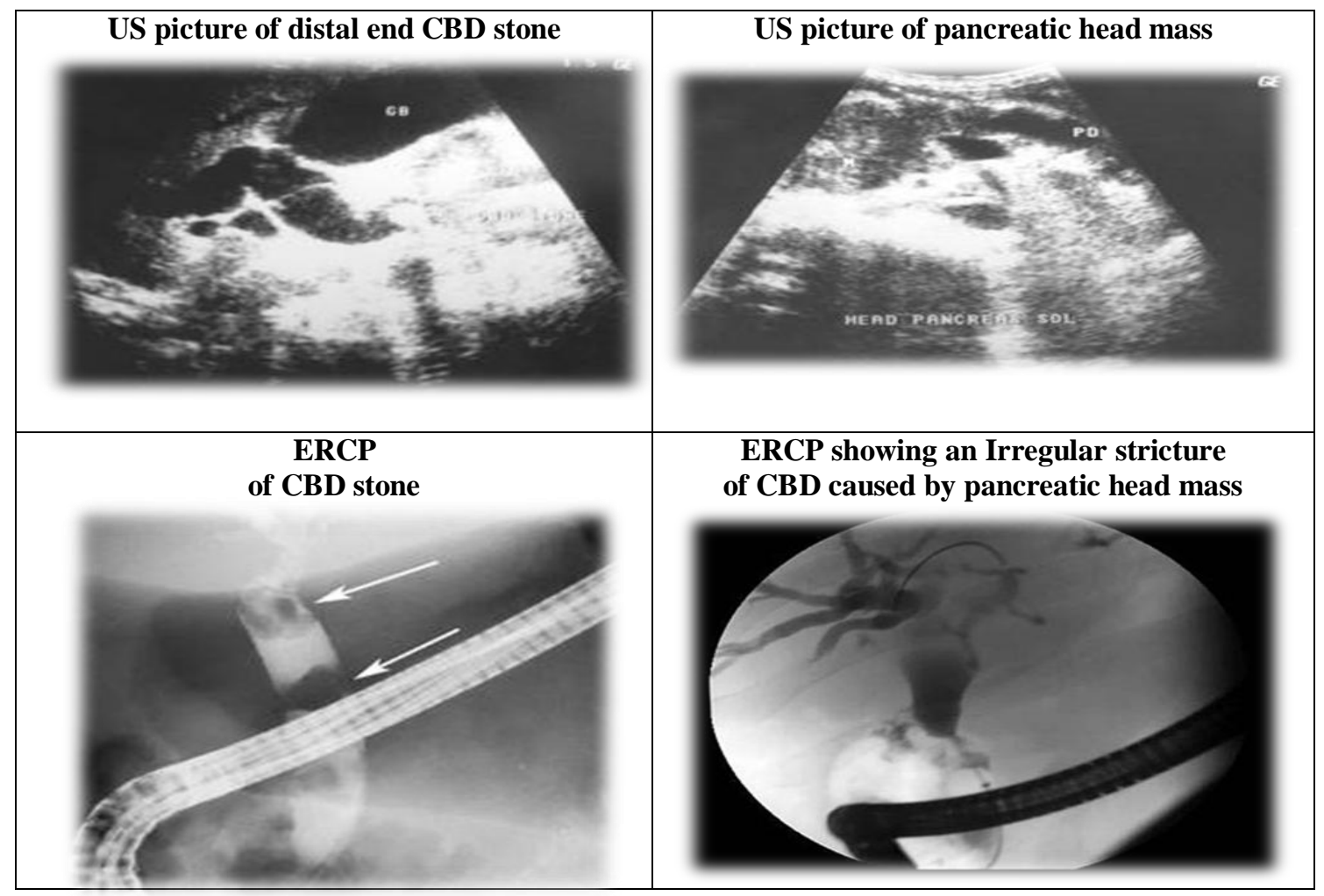

Salem and Sherbini, Afro-Egypt J Infect Endem Dis 2014; 4(1): 41-51 www.mis.zu.edu.eg/ajied/home.aspx 
Table (5): Relation between BMI and sex in cases with GB stones

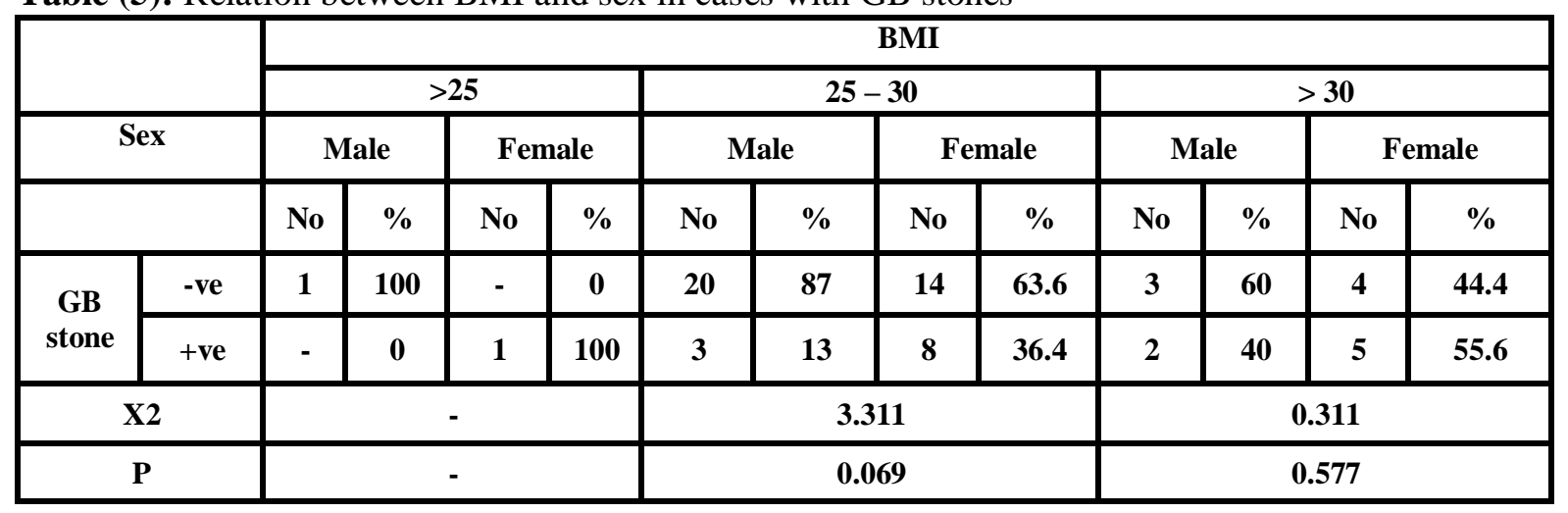

As shown in (Table 5) there was an increased percentage of females with BMI more than 30 in patients with GB stones, though it was non significant as $\mathrm{P}$ value was $>0.05$. Regarding the group with $\mathrm{BMI}<25$, there were only 2 patients (which was not sufficient for statistical analysis).

The sensitivity of U/S, CT and ERCP in detection of CBD stones was $76 \%, 88 \%$ and $100 \%$ respectively, whereas their sensitivity in detection of cancer head of pancreas was $66.7 \%$, $100 \%$ and $100 \%$ respectively. While the predictive values of US compared to ERCP in detection of CBD stones was $100 \%$ positive and $85.7 \%$ negative, and the predictive value of US compared to ERCP in detection of cancer head of pancreas was $100 \%$ positive and $94.5 \%$ negative. The predictive values of CT compared to ERCP in detection of CBD stones was $100 \%$ positive and $92.3 \%$ negative, while the predictive value of CT compared to ERCP in detection of cancer head of pancreas was $100 \%$ positive.

\section{DISCUSSION}

Among the many functions of the liver, production of bile is the most distinctive and liver specific. Adults produce approximately 500 $\mathrm{ml}$ of bile per day which is an aqueous solution containing bile salts, cholesterol proteins, bilrubin conjugates and others [5].

Cholestasis is caused by obstruction within the liver (intrahepatic) or outside the liver (extrahepatic). Intrahepatic cholestasis is characterized by widespread blockage of small ducts or by disorders, such as hepatitis, that impair the body's ability to eliminate bile. Extra-hepatic cholestasis results from the mechanical obstruction to large bile ducts outside the liver or within the porta hepatis [6].
This cross sectional study was performed to assess the frequency of cholestatic liver diseases and identify the frequency of extrahepatic causes as a health problem in our department. It was found that 61 out of 506 cases admitted to our tropical department had extraheaptic cholestasis representing $12.1 \%$. The high frequency of extraheaptic cholestasis in our study could be attributed to performing this study on a defined population, with high prevalence of chronic liver diseases and cirrhosis which increased the risk of gall bladder and common bile duct stones as noted by Marrelli [7] who studied the prevalence of calcular cholecystitis in Egyptian patients with chronic liver disease and found that chronic hepatitis $\mathrm{C}$ virus (HCV) infection is considered an important risk factor for the development of gallstone disease in those patients.

The lower incidence of obstructive jaundice in other studies as that reported by Khalili and Wilson [8] who reported its prevalence to be only 5 cases per 1000 people could be due to differences in the study group, as they assessed their incidence through a large scale study conducted on the general population. Also, the increased incidence of hepatocellular carcinoma in cases admitted to our department, could have contributed to the increased frequency in our study. The extra- hepatic cholestasis was more common among middle aged patients with a mean age of 51 years and a range of 25-72 years. This is in agreement with Bektas [9] who found almost similar results.

The majority of our patients had benign obstructive jaundice (57.4\%) while malignant causes comprised $(42.6 \%)$ which was in agreement with many other authors[10,11,12,13] who documented that the percentage of benign to malignant causes were about $60 \%-40 \%$. 
Choledocholithiasis was the most common cause among various causes of extrahepatic cholestasis in our study with a percentage of $40.9 \%$. Similar results were demonstrated by Khurram et al. [14] who stated that choledocholithiasis caused $35 \%$ of cases, being also the most common etiology of obstructive jaundice in their study. Moreover, in our study, patients with BMI $>30$ showed increased percentage of GB stones (55.6\%), while in those with BMI (25-30), the percentage was (36.4\%). Obesity and high dietary cholesterol could lead to increased biliary cholesterol secretion and decreased bile acid synthesis and pool which assist in cholesterol gall stone formation [21].

In our work extrahepatic cholestasis was more common in females $(54.1 \%)$, which was usually benign in nature $(60.6 \%)$ and common bile duct stone (CBD) obstruction as the most common cause in them $(42.2 \%)$. This may be due to the higher prevalence of cholelithiasis in females. Women are at greater risk of developing gall bladder cholesterol stones, which could be attributed to their estrogen levels. Many studies as Everson [17] have shown that, female steroid hormones can significantly alter hepatobiliary physiology. As the gall bladder volume increases during pregnancy or by the prolonged use of contraceptive pills and its emptying is delayed as well. Moreover, the biliary cholesterol content was increased and the bile acid metabolism was altered by the previous conditions.

Other benign causes in our study were acute pancreatitis $(6.6 \%)$, cholangitis $(3.3 \%)$, duodenal diverticulum, pancreatic pseudocyst and iatrogenic common bile duct (CBD) ligation with a percentage of $(1.6 \%)$ for each of them. This was not in agreement with Absi et al[18] who found Ascariasis and Hydatid disease of the billiary tract to be important benign causes of extrahepatic cholestasis, this difference could be attributed to the fact that their study was conducted in Saudi Arabia where high prevalence of these parasitic infections is present.

The percentage of pancreatitis and its complications as causes of extra-hepatic cholestasis, were higher in other studies done in USA and Europe by Granger and Remick, and Banks $[19,20]$. This could be explained by the high alcohol consumption in these countries predisposing to chronic pancreatitis.

The percentage of malignant extrahepatic cholestasis was higher in males $(50 \%)$ than females $(36.4 \%)$, being mainly cancer head of pancreas with reported incidence of $17.9 \%$. This is in agreement with Kiran and Pokola [21] who also found malignant obstructive jaundice to be more in males than females. Cancer head of pancreas had higher incidence with more mortality in males than females. This may be due to increased frequency of tobacco use in males which is considered a risk factor for that malignancy [22]. Similar observations were obtained by Syed et al.[10] and Khurram et al. [14] who found that the commonest causes of benign and malignant obstructive jaundice were cholidocholithiasis and cancer head of pancreas respectively .

Other malignant causes in our study included, ampullary carcinoma (13.11\%), cholangiocarcinoma (8.2\%), porta hepatis lymphadenopathy $(4.9 \%)$ and hepatocellular carcinoma (3.3\%). The results of Zarin et al.[23], was found to be similar to ours regarding malignant causes other than cancer head of pancreas.

Regarding the clinical manifestations, 58 cases (95.1\%) presented with jaundice. The other three cases were one patient diagnosed as ampullary carcinoma which is usually manifested by intermittent jaundice. Way [24] postulated that, the intermittent jaundice demonstrated in patients with ampullary carcinoma is attributed to tumor necrosis allowing passage of bile with transient relief of symptoms. While, the other two cases were manifested mainly with itching and upper abdominal pain. They were both diagnosed as CBD stone induced obstruction. Flores et al [25] mentioned that, CBD stones can lead to intermittent jaundice due to the bell and valve action induced by the stone at the lower end of CBD leading to partial and intermittent obstruction.

The percentage of dark urine, itching and clay stool in malignant cases were $92.3 \%, 73.1 \%$ and $53.8 \%$ respectively, compared to $82.8 \%, 62.8 \%$ and $48.6 \%$ respectively, in non malignant conditions. Charcot's triad was more common in benign conditions with a percentage of $(40 \%)$ compared to $(23.1 \%)$ in malignant cases. Sharma and Ahuja [26] said that dark urine and clay stool were more common in malignant obstructive jaundice while Charcot's triad was a more frequent presentation in benign conditions.

In selected cases in our study with extrahepatic cholestasis, the frequency of gall bladder (GB) stones was higher among cirrhotic patients $(52.9 \%)$ than non cirrhotics $(22.7 \%)$ with a 
significant relation between them. The pathophysiologic mechanisms responsible for induction of GB stones in cirrhotic patients may be related to altered bile pigments secretion, increased estrogen levels and/or abnormal gallbladder motility in cirrhosis[27]. The abnormal bile secretion in patients with liver cirrhosis may also be due to diminished liver reserve, damaged bile ductules, increased GB wall thickness caused by hyperemia and edema as well as decreased contractility with impaired emptying which could further contribute to gall stone formation [28].

In our study, there was a positive agreement between both GGT and ALP. This indicates that both markers together should be used for confirmation of cholestasis. The same finding was previously confirmed by [29].

According to imaging modalities ultrasound $(\mathrm{U} / \mathrm{S})$ was done to all cases. It picked dilated intrahepatic channels in $100 \%$ of patients of extrahepatic cholestasis and dilated CBD in $90.2 \%$ of them. CBD stones were detected in $31.1 \%$ while masses were detected in only $27.8 \%$ and most of the times, it was in the head of pancreas. The diagnostic accuracy of ultrasound was also studied by Akhtar and Mufti [30] and was close to our results as it was shown to be $85 \%$.

Abdominal computed tomography (CT) was done to all cases to confirm the diagnosis of $(\mathrm{U} / \mathrm{S})$ and/or to establish the cause of obstruction when U/S couldn't detect it. It picked dilated intrahepatic channels in $100 \%$ \& dilated CBD in $90.2 \%$, while CBD stones were detected in $36.1 \%$. Masses were detected in $41 \%$. It was superior to ultrasound in detection and localization of masses. It could detect 6 out of 8 cases with ampullary masses and all the 9 cases with pancreatic masses compared to zero and 6 cases respectively detected by ultrasound. CT could also detect the 4 cases with acute pancreatitis while U/S couldn't detect any of them. The higher efficacy of CT in diagnosis and staging of tumors causing obstructive jaundice has also been reported by Pasanen, et al. [31].

Endoscopic retrograde cholangiopancreatography (ERCP) was done to the cases which were not diagnosed by US or CT or as a therapeutic tool. It could detect all the 25 cases with CBD stones representing $40.9 \%$ of all cases with extrahepatic cholestasis. Cholangiocarcinoma was detected in 5 cases, in which 3 of them showed hilar CBD malignant stricture "Klatskin tumor" and 2 cases showed distal CBD stricture. Porta-hepatis lymphadenopathy and HCC were demonstrated as either hilar or distal malignant CBD stricture.

The sensitivity of US, CT and ERCP in detection of the various causes of extrohepatic obstruction was $76 \%, 88 \%$ and $100 \%$ respectively. These findings are broadly in agreement with studies done at various other centers for CT [31] and ERCP [32].

We can conclude that, the frequency of cholestatic liver diseases was $19.7 \%$, among them; extrahepatic causes comprised $12.1 \%$ in our department. Ultrasonography is considered the best primary non invasive diagnostic modality for extrahepatic cholestasis. While CT scans has more accuracy in detection of biliary and pancreatic masses and ERCP, is considered the gold standard modality in diagnosis and treatment of extrhepatic cholestasis with excellent accuracy in identification of the cause.

Funding: Non.

Conflicts of interest: The authors declare no conflict of interest.

Ethical approval: Informed consent was obtained from all participants, and the study was approved by the Ethical committee of Faculty of Medicine, Zagazig University.

\section{REFERENCES}

1. Chazouille'res $\mathrm{O}$ and Housset $\mathrm{C}$. Intrahepatic cholestasis. In: Rode's J, editor. Textbook of hepatology : from basic science to clinical practice. Oxford: Blackwell;2007 p. 1481-1500.

2. Heathcote EJ . Diagnosis and management of cholestatic liver disease. Clin Gastoentrol Hepatol 2007; 5: 776-782.

3. Dubrac S, Lear SR, Ananthanarayanan M, Balasubramaniyan N, Bollineni J, Shefer

$\mathrm{S}$ et al. Role of CYP27A in cholesterol and bile acid metabolism. J Lipid 2005; Jan;46(1):76-85.

4. Lee JG . Diagnosis and management of acute cholangitis. Nat Rev Gastroenterol Hepatol 2009 Sep; 6(9): 533-41.

5. Roche SP and Kobos R . Jaundice in the adult patient. American Family Physician 2004; 69 (2):299-304.

6. Mehrdad M, Seyed AM, Mohammad Taghi MS. Obstructive jaundice in Iran: factors affecting early outcome. Hepatobiliary Pancreat Dis Int 2008; 7:516-9. 
7. Marrelli D, Caruso S, Pedrazzani C, Neri A, Fernandes E, Marini M et al. CA19-9 serum levels in obstructive jaundice: clinical value in benign and malignant conditions. Am J Surg 2009; 198:333-9.

8. Khalili, K, Wilson, SR . The biliary tree and gallbladder. In: Rumack CM, Wilson SR, Charboneau JW, et al, editors. Diagnostic ultrasound. St Louis, MO: Mosby2005; p.171212.

9. Bektas, M, Dokmeci, A, Cinar, K, Halici I, Oztas E, Karayalcin S et al. Endoscopic management of biliary parasitic Diseases. Dig Dis Sci 2010 ;55:1472-1478.

10. Syed, N.; Mohammad, SA.; Umair, UI.; et al. Etiological spectrum of obstructive jaundice .Medical channel 2010; 16:299-301.

11. EASL (European Association for Study of the Liver ). Management of cholestatic liver diseases. J Hepatol 2009; 51: 237-267.

12. Steinberg, W. The clinical utility of the CA 19-9 tumor associated antigen. Am J Gastroenterol1999; 85 (4): 350-355.

13. Iqbal J, Khan Z, Afridi FG et al. Causes of obstructive jaundice. Pakistan J Surg 2008; 14(1):12-14.

14. Khurram, M, Durrani, AA; Hasan, $Z$ et al. Endoscopic retrograde cholangiopancreatographic evaluation of patients with obstructive jaundice. J Coll Physicians Surg Pak 2009; 13:325-8.

15. Bekele, Z and Yifru A . Obstructive jaundice in adult Ethiopians in a referral hospital. Ethiop Med J 2010; 38:267-75.

16. Siddique K, Ali Q, Mirza S, Jamil A, Ehsan A, Latif S, Malik AZ. Evaluation of the aetiological spectrum of obstructive jaundice. J Ayub Med Coll Abbottabad 2008; 20:62-66.

17.Everson GT, McKinley C, F Kern Jr. Mechanisms of gallstone formation in women. Effects of exogenous estrogen (Premarin) and dietary cholesterol on hepatic lipid metabolism. J Clin Invest 2011; 87(1): 237-246.

18. Absi, MA.; Qais, AM., Katta . Billiary Ascariasis, the value of Ultrasound in the diagnosis and management. Ann Saudi Med 2007; 27 (3) : 161163

19. Granger J , Remick D . Acute pancreatitis: models, markers, and mediators. Shock2005; 24 Suppl 1:45-51.
20. Banks PA . Epidemiology, natural history, and predictors of disease outcome in acute and chronic pancreatitis. Gastrointest Endosc2002; 56 (6 Suppl): S226-30.

21. Kiran, RP , Pokola N . Bile duct tumors. Emedicine. Available from: URL: 2001 http://www.emedicine.com/med/topic 2705.htm.

22. Zhang J, Dhakal,I., Yan H Phillips M, Kesteloot $\mathrm{H}$; SEER Cancer Registries . Trends in pancreatic cancer incidence in nine SEER Cancer Registries, 1973-2002. Ann Oncol 2007; 18:1268-79.

23. Zarin, M, Ahmed, M, Gohar, A et al. Incidence of gall stones in carcinoma gall bladder patients . Pak j Surg 2005; 21:19- 22.

24. Way LW . Biliary tract. In Way LW (ed). "Current surgical diagnosis and treatment." 2004 Norwalk: Appleton and Lange 1994; p.537-566.

25. Flores, C, Maguilnik, I, Hadlich, E ,Goldani LZ. Microbiology of choledochal bile in patients with choledocholithiasis admitted to a tertiary hospital. J Gastroenterol Hepatol 2003; 18:333-46.

26. Sharma, MP and Ahuja, VX (1999): Aetiological spectrum of Obstructive Jaundice and the diagnostic ability of ultrasonography: A clinician's perspective. Trop Gastroenterol 1999; 20: 167-9.

27. Shaffer EA . Gallstones disease: Epidemiology of gallbladder stone disease. Best Pract Res Clin Gastroenterol 2006; 20:981-996.

28. Acalovschi, M, Dumitrascu, D and Nicoara, C Gallbladder contractility in liver cirrhosis: comparative study in patients with and without gallbladder stones. Dig Dis Sci 2009; 49:17-24.

29. Kern, F Jr. Effects of dietary cholesterol on cholesterol and bile acids homeostasis in patients with cholesterol gallstones. J Clin Invest 2004; 93:1186-94.

30. Akhtar, S, Mufti TS . Diagnostic accuracy of obstructive jaundice on ultrasono-graphu at Ayub Hospital complex. J Ayub Med Coll Abottabad 2009;11:45-6.

31. Pasanen PA, Partanen K, Pikkarainen P Alhava E, Pirinen A, Janatuinen E. Diagnostic accuracy of ultrasound, computed tomography and endoscopic retrograde cholangiopancreatography in the detection of obstructive jaundice. Scand $J$ Gastroenterol 1991; 26(11): 1157-64.

32. Hekimoglu K . MRCP vs. ERCP in the evaluation of biliary pathologies: review of current literature. J Dig Dis. Aug 2008; 9(3):162-9. 
Peer reviewer: Amira Suliman;Professor of Tropical Medicine and Hepatogastroenterology Faculty of Medicine ,Zagazig University,Egypt.Editor: Tarik Zaher;
Professor of Tropical Medicine and Hepatogastroenterology Faculty of Medicine, Zagazig University,Egypt. 